\title{
IATROGENIC UROLOGICAL INJURIES IN PELVIC SURGERIES, OUR INSTITUTIONAL EXPERIENCE- A DESCRIPTIVE STUDY
}

\author{
Senthilvel Arumugam¹, Periasamy Ponnusamy2, Amritha Balakumar ${ }^{3}$
}

1 Professor, Department of Urology, Government Kilpauk Medical College Hospital, Chennai, Tamilnadu.

2Professor, Department of Urology, Government Kilpauk Medical College Hospital, Chennai, Tamilnadu.

${ }_{3}^{3}$ MCh Postgraduate Student, Department of Urology, Government Kilpauk Medical College Hospital, Chennai, Tamilnadu.

\begin{abstract}
\section{BACKGROUND}

Ureteric and bladder injury is a serious concern during pelvic surgery and represents one of the most dreaded complications during gynaecologic operations with an overall incidence ranging from $0.5 \%$ to almost $30 \%$. The rate of ureteral injury is increased when technically demanding laparoscopic and radical pelvic surgeries.

Aims and Objectives- To study the incidence of ureter and bladder injuries detected intraoperatively/ postoperatively following pelvic surgeries in the gynaecology and oncology departments of our institution. This study describes our experience in the management of ureteric/ bladder injuries following pelvic operations and outcome of management of this condition in our local setting.
\end{abstract}

\section{MATERIALS AND METHODS}

This is a retrospective descriptive study of patients with iatrogenic injuries to the ureter and bladder following pelvic surgeries that were managed in our department from June 2016 to January 2018. Analysis of the patient's diagnosis, indication for surgery, type of surgery, time at diagnosis of injury, presenting features and the type of management was done.

\section{RESULTS}

A total of 9 ureteric injuries and 6 bladder injuries were identified out of 293 pelvic surgeries performed in the study period. All bladder injuries were diagnosed and managed intraoperatively. 80\% of ureteric injuries were diagnosed postoperatively with fever, flank pain and leakage of urine being the most common presentation. Duration of symptoms ranged from immediate post-op period to up to 6 months after surgery. $45 \%$ were diagnosed with ureteric stricture, $33 \%$ with ureterovaginal fistula and $22 \%$ were diagnosed intraoperatively. Incidence of ureteric injuries in simple hysterectomy for benign causes was $1.8 \%$ and for Wertheim's hysterectomy for malignancy was $6 \%$. Incidence of ureteric injuries following laparoscopic hysterectomy was 7\%. Ureteric injury following APR was found to be $7 \%$. We performed ureteroneocystostomy for 4 cases, Boari flap for 2 cases, transureteroureterostomy in 1 case, nephrectomy in 1 case and ureterocolonic anastomosis in 1 patient. Postoperative complications observed were surgical site infection in $33 \%$ and urosepsis in $11 \%$ of patients. Mortality was nil.

\section{CONCLUSION}

Laparoscopic and Wertheim's hysterectomy were most commonly associated with ureteric injuries. Meticulous surgical technique as well as identification of the course of the ureter and associated anatomic locations where injury is most likely to occur is important to decrease the risk of ureteric injury. Timely recognition of ureteric injury and its management is associated with good outcome.

\section{KEY WORDS}

Ureteric and Bladder Injuries, Iatrogenic, Abdomino-Pelvic Operations, Management, Outcomes.

HOW TO CITE THIS ARTICLE: Arumugam S, Ponnusamy P, Balakumar A. Iatrogenic urological injuries in pelvic surgeries, our institutional experience- a descriptive study. J. Evolution Med. Dent. Sci. 2018;7(21):2588-2591, D0I: 10.14260/jemds/2018/583

\section{BACKGROUND}

Ureteric and bladder injury is a serious concern during pelvic surgery and represents one of the most dreaded complications during gynaecologic operations with an overall incidence ranging from $0.5 \%$ to almost $30 \% .{ }^{1}$ The rate of ureteral injury is increased when technically demanding laparoscopic and radical pelvic surgeries.

'Financial or Other Competing Interest': None.

Submission 10-04-2018, Peer Review 05-05-2018,

Acceptance 12-05-2018, Published 21-05-2018.

Corresponding Author:

Dr. Senthilvel Arumugam,

Professor,

Department of Urology, Surgical Block $5^{\text {th }}$ Floor,

Kilpauk-600010, Kilpauk Medical College Hospital,

Chennai, Tamilnadu, India.

E-mail: arusenthil2014@gmail.com

DOI: $10.14260 /$ jemds $/ 2018 / 583$
Ureteral injury may be recognised intraoperatively and treated without any complications or it may be overlooked and present in the immediate or late postoperative period leading to substantial morbidity, threatening kidney viability and justifying medico-legal litigation. Surgical reconstruction constitutes the mainstream therapy of choice for patients presenting with symptoms suggestive of ureteral obstruction in the postoperative period. These procedures include endto-end ureteroureteral anastomosis, ureteral reimplantation, ureteroneocystostomy or some combination. ${ }^{2}$

The risk factors for iatrogenic ureteric injury include nature and indication of the abdominal or pelvic surgery, patient related factors such as pelvic adhesions from previous surgeries, history of pelvic radiation, enlarged uterus, pelvic malignancy, pelvic endometriosis and anatomical abnormalities..$^{3,4}$ Inexperience of the operating surgeon may also be an important risk factor. During abdomino-pelvic surgery ureteric injury may be in the form of crush injury by 
a clamp, inadvertent diathermy injury, suture ligation, transaction, resection of a segment of ureter, kinking of ureter and devascularisation of a segment of ureter due to extensive dissection close to ureter. Patient may develop urinary fistula with adjacent organ or end up with nonfunctioning kidney. The most important determinant of outcome of ureteric injuries is the interval between the injury and repair: the longer the interval, the worse the outcome. 5

Prompt intraoperative identification and appropriate correction decreases morbidity and eliminate mortality. However, most cases of ureteric injuries are recognised late. Patients may present with flank pain, fever, prolonged ileus, ascites, urinary incontinence, anuria and azotaemia with 5\% patients presenting very late with hydronephrosis and nonfunctioning kidney. The objective of the study was to describe our experiences in the management and outcome of iatrogenic ureteric injuries, highlighting the causes, clinical presentation and treatment outcome of iatrogenic ureteric injuries in our setting.

\section{MATERIALS AND METHODS}

This retrospective descriptive study analyses iatrogenic urological injuries managed in the Department of Urology, KMC, Chennai between June 2016 and January 2018. Informed consent was obtained from all patients to analyse their data.

All patients presenting to our department postoperatively and intraoperative call overs for urological injuries during pelvic surgeries were included in the study. Patient's symptoms, time duration to presentation, type and technique of pelvic surgery performed, indication for the procedure, any risk factors like bulky uterus ( $>12 \mathrm{wks}$ ), endometriosis, prior pelvic radiation and surgeries like C-section were noted. Nature of injury and its sequelae were diagnosed by abdomino-pelvic scans and CECT with urogram and corrected surgically with appropriate procedures. Outcomes of management were measured by length of hospital stay, complications and mortality. Data were analysed in terms of frequency and percentage using Microsoft Excel 2007.

\section{RESULTS}

A total of 293 patients underwent pelvic surgeries in our hospital during our study period, out of which 9 patients had ureteric injury and 6 patients had bladder injury. All the bladder injuries and 2 ureteric injuries were identified intraoperatively, and primary repair was performed.

Most common procedure associated with ureteric and bladder injuries was hysterectomy. Incidence of ureteric injuries with simple hysterectomy was $1.8 \%$ and for Wertheim's hysterectomy was $6 \%$. Incidence with laparoscopic surgery was $7.4 \%$ and with open technique was $2 \%$. Incidence of bladder injury was $2.14 \%$ and was associated with hysterectomy. Abdominoperineal resections were associated with $7.6 \%$ of ureteric injuries.

\begin{tabular}{|c|c|c|}
\hline Surgery & Frequency & Percentage \\
\hline TAH with BSO & 165 & $1.8 \%$ \\
\hline Wertheim's hysterectomy & 82 & $6 \%$ \\
\hline Abdominoperineal resection & 13 & $7 \%$ \\
\hline \multicolumn{2}{|c|}{ Table 1. Aetiology in Ureteric Injuries } \\
\hline
\end{tabular}

\begin{tabular}{|c|c|c|}
\hline Variable & Frequency & Percentage \\
\hline Laparoscopy & 54 & $7.4 \%$ \\
\hline Open surgery & 178 & $2.24 \%$ \\
\hline \multicolumn{2}{|r|}{ Table 2. Surgery Characteristics }
\end{tabular}

Known risk factors for ureteric injuries were observed in $77.7 \%$ of the patients.

\begin{tabular}{|c|c|c|}
\hline Variable & Frequency & Percentage \\
\hline Prior pelvic surgery & 89 & $31.7 \%$ \\
\hline Prior pelvic radiation & 56 & $19 \%$ \\
\hline $\begin{array}{c}\text { Adverse uterine pathology like } \\
\text { cancer, bulky uterus, endometriosis } \\
\text { etc. }\end{array}$ & 84 & $30 \%$ \\
\hline \multicolumn{2}{|c|}{ Table 3. Risk Factors } \\
\hline
\end{tabular}

Out of these patients, one patient had obstructed nonfunctioning kidney. $11 \%$ were diagnosed with bilateral ureteric stricture with oliguria and azotaemia. 44\% patients had ureteric stricture, $33 \%$ patients were diagnosed with ureterovaginal fistula. Distal ureter was the most frequently involved site in $77 \%$ of cases. Suture ligation was observed in open technique and thermal injury in laparoscopic surgery.

\begin{tabular}{|c|c|c|}
\hline Variable & Frequency & Percentage \\
\hline Intraoperatively detected & 2 & $22 \%$ \\
\hline Postoperative diagnosis & 7 & $77 \%$ \\
\hline Unilateral-right & 6 & $66 \%$ \\
\hline Unilateral-left & 2 & $22 \%$ \\
\hline Bilateral & 1 & $11 \%$ \\
\hline Mid-ureter & 2 & $22 \%$ \\
\hline Distal ureter & 7 & $77 \%$ \\
\hline Ureteric stricture & 4 & $44 \%$ \\
\hline Ureterovaginal fistula & 3 & $33 \%$ \\
\hline \multicolumn{2}{|c|}{ Table 4. Injury Characteristics } \\
\hline
\end{tabular}

Most common presenting symptom was fever observed in $55 \%$ of patients followed by flank pain in $44 \%$, urine leak through vagina in $33 \%$, azotaemia in $22 \%$ and haematuria in $11 \%$ of patients with most symptoms overlapping.

\begin{tabular}{|c|c|c|}
\hline Variable & Frequency & Percentage \\
\hline Fever & 5 & $55 \%$ \\
\hline Loin pain & 4 & $44 \%$ \\
\hline Azotaemia & 2 & $22 \%$ \\
\hline Urine leak through vagina & 3 & $33 \%$ \\
\hline Haematuria & 1 & $11 \%$ \\
\hline Non-functioning kidney & 1 & $11 \%$ \\
\hline \multicolumn{2}{|c|}{ Table 5. Patient Presentation } \\
\hline
\end{tabular}

Pre-operative assessment included Ultrasound scan, Intravenous urography (IVU), Computed tomography (CT) scan, examination under anaesthesia, dye test and cystoscopy as indicated. All the injuries were repaired as soon as possible after diagnosis was made and stabilisation of their condition done. Ureteroneocystostomy was the most frequent reconstructive surgery performed in $45.0 \%$ of cases.

\begin{tabular}{|c|c|c|}
\hline Surgery & Frequency & Percentage \\
\hline Ureteroneocystostomy & 4 & $44 \%$ \\
\hline Boari flap & 2 & $22 \%$ \\
\hline Transureteroureterostomy & 1 & $11 \%$ \\
\hline Nephrectomy & 1 & $11 \%$ \\
\hline Ureterocolonic anastomosis & 1 & $11 \%$ \\
\hline \multicolumn{3}{|c|}{ Table 6. Procedures Performed for Ureteric Injuries } \\
\hline
\end{tabular}




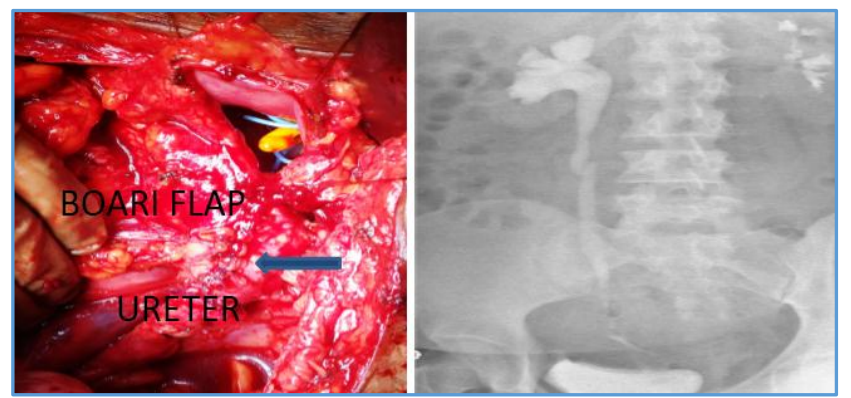

Figure 1. Boari Flap

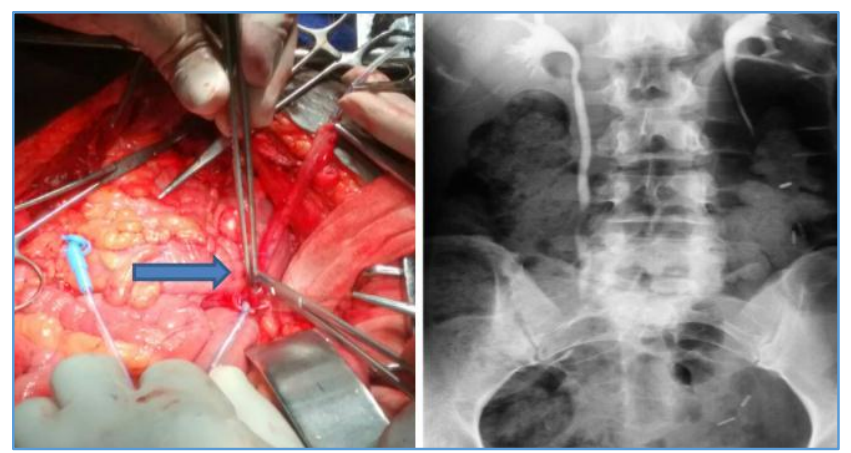

Figure 2. Transureteroureterostomy

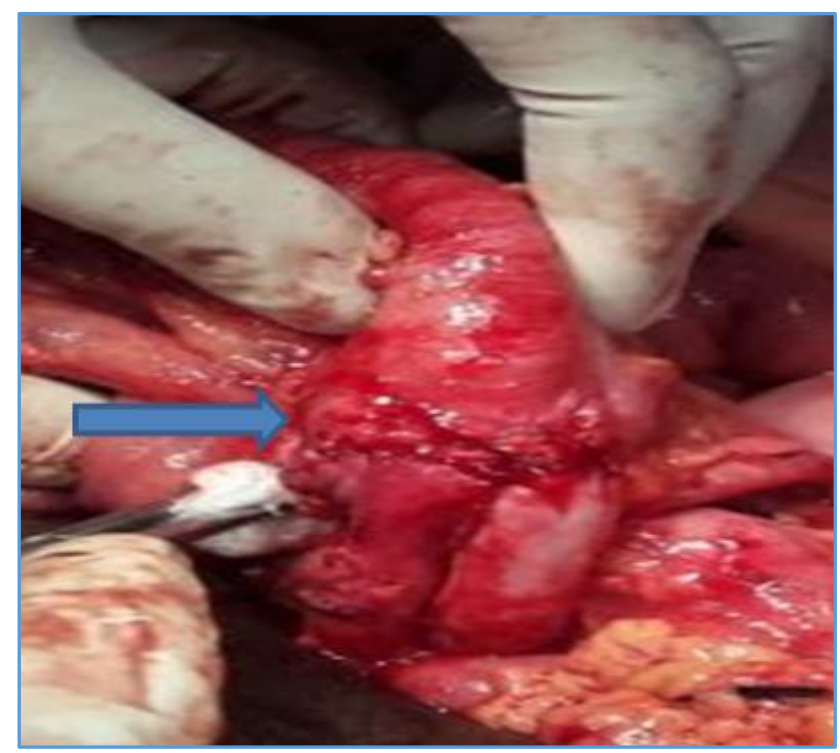

Figure 3. Ureterocolonic Anastomosis

Postoperative complications observed were surgical site infections in $33 \%$ and urosepsis in $11 \%$. Mortality in this group of patients was nil. Patients were followed up for a period ranging between two and twelve months. Follow-up evaluation mainly consisted of clinical evaluation and abdomino-pelvic ultrasound scan with IVU and cystoscopy when indicated.

\section{DISCUSSION}

Iatrogenic injury to the ureter is the most common complication of abdomino-pelvic surgery, ranging from less than 1 to 10 percent of procedures depending on the complexity of the procedure. ${ }^{6}$

\begin{tabular}{|c|c|c|}
\hline Surgery & RCOG & Our Study \\
\hline Abdominal hysterectomy & $0.4-3 \%$ & $2.24 \%$ \\
\hline Wertheim's hysterectomy & $1-30 \%$ & $6 \%$ \\
\hline Laparoscopic hysterectomy & $0.2-6 \%$ & $7.4 \%$ \\
\hline Colorectal surgery & $0.15-10 \%$ & $7 \%$ \\
\hline \multicolumn{3}{|c|}{ Table 7. Incidence of Ureteric Injuries } \\
\hline
\end{tabular}

In this study the incidence of ureteric injury following pelvic surgeries was $3.07 \%$, a figure comparable to those in the literature. ${ }^{2}$ Surgeries performed for malignancy like Wertheim's hysterectomy and abdomino-perineal resections had high incidence of ureteric injuries- $6 \%$ and $7 \%$ respectively. Laparoscopic hysterectomy had a higher incidence of injuries than open transabdominal procedure.

In our series gross pelvic adhesions from previous surgeries, previous history of pelvic inflammatory diseases and massive intraoperative bleeding during operations for large pelvic tumours were reported to be risk factors for iatrogenic ureteric injuries. ${ }^{7}$

Most ureteric injuries occur in the distal most part of the ureter where it is closely related to the uterine vessels. ${ }^{4,8}$ In this study $77 \%$ injuries occurred in the distal portion of the ureter, which is in agreement with other studies. The distal portion of the ureter is not only embryological related to the female genital organs, but is also involved in diseases affecting them.9,8 At the base of the cardinal ligament where it crosses the uterine artery, the ureter is just $12 \mathrm{~mm}$ from the vagina and as it moves towards the bladder it becomes even closer to the vagina and this predisposes the ureter to injury during surgical procedures in the pelvis.

Bilateral ureteric injuries have been reported in literature to occur in $5-10 \%$ of patients. In this study, the incidence was noted to be $11 \% .8,10$ The left ureter has a greater proximity to the cervix compared to the right ureter and is thus more liable to injury; however, in our study right ureter was most commonly injured. 11

Imaging is essential for diagnosis, especially in cases where bilateral lesions are suspected. In this study preoperative assessment included Ultrasound scan, Intravenous urography (IVU), Computed tomography (CT) scan, examination under anaesthesia, dye test and cystoscopy as indicated.12,10

Many authors have suggested that early recognition and management of ureteric injuries is associated with better outcome.2,13 Intraoperative diagnosis was made in only $22 \%$ of the patients and $77 \%$ were identified postoperatively. Missed injury with presentation of non-functioning kidney was seen in $11 \%$ of patients. ${ }^{8}$

In the present study, percutaneous nephrostomy was performed in only 2 patients representing $22 \%$ of cases. ${ }^{8}$ However, nephrostomy tube in this study was used only to stabilise the patients and all of these patients required additional definitive procedures.

Various reconstructive surgical options include ureteroneocystostomy, Boari flap, Psoas hitch, end-to-end ureteroureterostomy and trans-ureteroureterostomy. Other options include ileal segment replacement, appendix interposition and auto-transplantation. ${ }^{2}$

In our study, ureteroneocystostomy was the most common procedure performed. Boari flap repair was done in 2 patients and transuretero-ureterostomy, nephrectomy and 
ureterocolonic anastomosis were performed in one patient each respectively. All patients were stented.

In our study, the most common complication was surgical site infection seen in $22 \%$ patients followed by urosepsis. All patients recovered well in the postoperative period. Mortality in the study group was nil. ${ }^{8}$

The outcome of our management was generally good with a $92.7 \%$ success rate and no complications that were detected during the follow-up period. This may be related to the fact that all reconstructive procedures were performed by experienced urologists.

The potential limitation of this study is its retrospective nature with incomplete data regarding the detailed operative procedure and postoperative management of the primary surgery.

\section{CONCLUSION}

Our experience in this study shows that iatrogenic ureteric injuries are still common in our environment and total abdominal hysterectomy accounts for most cases. The majority of injuries are a result of complications of abdomino-pelvic operations by general practitioners in the peripheral hospitals. Meticulous surgical technique as well as identification of the course of the ureter and associated anatomic locations where injury is most likely to occur is important to decrease the risk of ureteric injury. Early recognition and prompt repair of ureteric injuries is the key to a successful outcome. Treatment of these injuries by experienced team may minimise long-term consequences.

\section{REFERENCES}

[1] Obarisiagbon EO, Olagbuji BN, Onuora VC, et al. Iatrogenic urological injuries complicating obstetric and gynaecological procedures. Singapore Med J 2011;52(10):738-41.

[2] Al-Awadi K, Kehinde EO, Al-Hunayan A, et al. Iatrogenic ureteric injuries: incidence, aetiological factors and the effect of early management on subsequent outcome. Int Urol Nephrol 2005;37(2):235-41.
[3] Chou MT, Wang CJ, Lien RC. Prophylactic ureteral catheterization in gynaecologic surgery: a 12-year randomized trial in a community hospital. Int Urogynecol J Pelvic Floor Dysfunct 2009;20(6):68993.

[4] Kim JH, Moore C, Jones JS, et al. Management of ureteral injuries associated with vaginal surgery for pelvic organ prolapse. Int Urogynecol J Pelvic Floor Dysfunct 2006;17(5):531-5.

[5] Mteta KA, Mbwambo J, Mvungi M. Iatrogenic ureteric and bladder injuries in obstetric and gynaecological surgeries. East Afr Med J 2006;83(2):79-85.

[6] Preston JM. Iatrogenic ureteric injury: common medicolegal pitfalls. BJU Int 2000;86(3):313-7.

[7] Oboro VO, Dare FO, Fadiora SO, et al. Ureteric injuries following pelvic operations. East Afr Med J 2002;79(11):611-3.

[8] Tijani KH, Onwuzurigbo KI, Ojewola RW, et al. Iatrogenic ureteric injuries in a Nigerian teaching hospital: experience in the last decade. East Afr Med J 2011;88(9):304-9.

[9] Liapis A, Bakas P, Giannopoulos V, et al. Ureteral injuries during gynecological surgery. Int Urogynecol J Pelvic Floor Disfunct 2001;12(6):391-4.

[10] Chinakwana GU, Okafor PI, Ikechebelu JI, et al. Urological injuries following gynaecological operations - our experience in a teaching hospital in Nigeria. West Afr J Med 2006;25(2):153-6.

[11] Fröber R. Surgical atlas, surgical anatomy of the ureter. BJU Int 2007;100(4):949-65. doi: 10.1111/j.1464-410X.2007.07207.x.

[12] Vakili B, Chesson RR, Kyle BL, et al. The incidence of urinary tract injury during hysterectomy: a prospective analysis based on universal cystoscopy. Am J Obstet Gynecol 2005;192(5):1599-604.

[13] Williams G, Broughton S, Worku H, et al. Five years' experience of ureterovaginal fistulae following obstetric or gynaecological intervention in Ethiopia. Afr J Urol 2010;16(1):17-9. 